\title{
Hormonal influences on neuroimmune responses in the CNS of females
}

\author{
Nela Monasterio ${ }^{1}$, Edgar Vergara ${ }^{2}$ and Teresa Morales ${ }^{1}$ * \\ ' Departamento de Neurobiología Celular y Molecular, Instituto de Neurobiología, Universidad Nacional Autónoma de México, Santiago de Querétaro, México \\ 2 Departamento de Biología, Facultad de Química, Universidad Nacional Autónoma de México, Ciudad de México, México
}

\section{Edited by:}

Beatriz Gomez-Gonzalez, Universidad

Autónoma Metropolitana - Unidad

Iztapalapa, Mexico

Reviewed by:

Beatriz Gomez-Gonzalez, Universidad Autónoma Metropolitana - Unidad

Iztapalapa, Mexico

George Chrousos, National and

Kapodistrian University of Athens,

Greece

\section{*Correspondence:}

Teresa Morales, Departamento de

Neurobiología Celular y Molecular, Instituto de Neurobiología,

Universidad Nacional Autónoma de

México, Boulevard Juriquilla 3001 ,

76230, Santiago de Querétaro,

México

e-mail:marter@unam.mx
Particular reproductive stages such as lactation impose demands on the female. To cope with these demands, her physiology goes through numerous adaptations, for example, attenuation of immune and stress responses. Hormonal fluctuation during lactation exerts a strong influence, inducing neuroplasticity in the hypothalamus and extrahypothalamic regions, and diminishing the stress and inflammatory responses. Thus, hormones confer decreased vulnerability to the female brain. This mini-review focuses on the adaptations of the immune and stress response during maternity, and on the neuroprotective actions of progesterone and prolactin and their effects on inflammation. The importance of pregnancy and lactation as experimental models to study immune responses and disease is also highlighted.

Keywords: stress, progesterone, prolactin, lactation, neuroimmune, HPA axis, female

\section{INTRODUCTION}

Reproduction is one of the most significant events in the life of a mammalian female. It can be described as a rich social and hormonal experience that begins by interacting and mating with a male, followed by pregnancy, parturition, and ultimately lactation, interaction with pups, and weaning (Mann and Bridges, 2001; Serafim and Felicio, 2002). Striking behavioral and neuroendocrine alterations due to motherhood have been reported in several mammalian species including mice, rats, rabbits, sheep, and humans. They are reflected by changes at almost all brain levels and are essential to protect the developing embryo, and for successful delivery, maternal behavior, and pup survival (Kinsley and Lambert, 2008). These neuroanatomical and functional changes observed during pregnancy and lactation are necessary to cope with the demands of reproduction and to protect the maternal organism against dramatic hormonal variations (Mann and Bridges, 2001; Kinsley and Lambert, 2008). They include a marked adaptation of the hypothalamus-pituitary-adrenal (HPA) axis, which results in hypo-responsiveness to stress, diminished inflammatory responses (Walker et al., 1992; Windle et al., 1997a; Lightman et al., 1998; Tilbrook and Clarke, 2006), and diminished sensitivity of the brain of the mother against excitotoxic damage (Morales, 2011).

\section{MECHANISMS GOVERNING THE HYPO-RESPONSIVENESS TO STRESS DURING PREGNANCY AND LACTATION}

One of the best-known examples of naturally attenuated stress response is seen in the female rat during late pregnancy and lactation (Tilbrook and Clarke, 2006). During these reproductive phases, the HPA axis maintains minimal responses necessary to cope with any situation that may threaten the homeostasis of the female. This axis comprises corticotropin-releasing hormone (CRH)- and vasopressin- neurons in the paraventricular nucleus of the hypothalamus (PVN), which when stimulated, release these peptides into the median eminence to stimulate pituitary cell production of adrenocorticotropic hormone $(\mathrm{ACTH})$ that reaches the adrenal cortex to release cortisol or corticosterone. HPA axis activity is regulated by glucocorticoid negative feedback on the pituitary, the PVN, and higher brain centers.

The attenuation of the stress response during pregnancy and lactation has been documented in various different stress models (Lightman et al., 2001; Russell etal., 2008). Pregnancy in the rat is accompanied by a progressive decrease in HPA axis responses to a range of psychological (Neumann et al., 1998) and physical (Brunton et al., 2005; Douglas et al., 2005) stressors particularly in the last week of pregnancy, reflected by reduced ACTH and corticosterone secretion. This hypo-responsiveness persists through parturition (Wigger et al., 1999; Neumann et al., 2003) and lactation until weaning (Windle et al., 1997b). Suppressed responses to stress in pregnancy can be explained by adaptations in both the anterior pituitary and the hypothalamus (Brunton et al., 2005; Russell et al., 2008). Corticotrophs in the pituitary are less sensitive to secretagogs (Shanks et al., 1997; Neumann et al., 1998), and CRH mRNA expression induced by stress is attenuated (Brunton etal., 2005). Moreover, HPA axis responses to immune stress in early mid pregnancy are strong and similar to that in virgins, although activation of hypothalamic vasopressin neurons, rather than CRH neurons, may be more important in 
the stress response in pregnancy (Ma et al., 2005; Parker et al., 2011).

Studies in lactating rats have shown a flattening of the diurnal rhythm of corticosterone secretion (Walker et al., 1992; Atkinson and Waddell, 1995) during this phase, such that the nadir levels of corticosterone rise (Stern et al., 1973; Fischer et al., 1995) and the peak evening levels decrease (Windle et al., 2013). During lactation, basal plasma concentrations of both ACTH and corticosterone increased in lactating animals compared with those in virgin rats (Shanks et al., 1997, 1999). There is also an increased basal expression of CRH mRNA in the PVN in early lactation (day 3; da Costa et al., 1996), but low basal expression of CRH mRNA by the middle phase of the lactation period (days 7-10; Windle et al., 1997a; Walker et al., 2001). However, CRH expression in response to stress is diminished at either stage (Lightman and Young, 1989; Windle et al., 1997b), similar to findings in late pregnancy (Douglas et al., 2005).

The mechanisms for this altered neuroendocrine responses include diminished CNS excitatory signaling within stressresponsive systems like the catecholaminergic brainstem and limbic circuitry (Hoffman etal., 1994; da Costa et al., 1996; Wintrip etal., 1997) as well as an altered hypothalamic and adrenomedullary catecholamine function (Patel et al., 1993; Toufexis and Walker, 1996; Windle et al., 1997a; Toufexis et al., 1998). Attenuated noradrenergic tone also underlies stress hyporesponsiveness in lactation, and is clearly dependent upon suckling (Hoffman et al., 1994; Toufexis and Walker, 1996; Toufexis et al., 1998). Moreover, opioids switch to having a net inhibitory effect on HPA activity such that naloxone treatment enhances the ACTH response to IL-1 $\beta$ in late pregnant rats (Brunton et al., 2009). Growing evidence for an integrated participation of many other factors besides noradrenaline and opioids that underlie altered responses to stress, include CRH (Johnstone et al., 2000; da Costa et al., 2001), oxytocin (Neumann et al., 2003; Windle et al., 2004), prolactin (PRL; Torner and Neumann, 2002), steroids and neurosteroids (Douglas et al., 2000; Brunton and Russell, 2008, 2011; Figure 1).

Another mechanism for the attenuation of the stress response involves nitric oxide (NO) regulation of $\mathrm{CRH}$ expression within the PVN. NO is present in the PVN of female rats during lactation (Popeski et al., 1999; Otukonyong et al., 2000; Monasterio and Morales, 2011), as indicated by the NO markers NADPHdiaphorase and neuronal synthase (nNOS), and its synthesis dependent on suckling (Otukonyong et al., 2000) and PRL (Vega et al., 2010). These markers increase within the PVN in response to stress in male rats, but paradoxically, basal level of NO markers are present in the PVN of lactating females whose response to stress is attenuated. The activational c-fos response and an increase in NADPH-diaphorase- and nNOS-positive cells are clearly detected in the PVN of diestrus rats after an immune challenge, but not in lactating rats. Furthermore, the total concentration of nitrates in the hypothalamus and the circulating level of corticosterone and IL-6 increase significantly after stress in diestrus, but not in lactating rats, compared to their corresponding controls. Intracerebral administration of L-NAME, a general NOS inhibitor, reverses the attenuation of the activational response to stress in the lactating rats and increases $\mathrm{CRH}$ expression, suggesting that sustained NO production in the PVN during lactation contributes to attenuate the neuroendocrine response to stress (Monasterio and Morales, 2011; Figure 1).

\section{HPA AXIS HORMONES, PROGESTERONE, AND PROLACTIN IN THE REGULATION OF THE IMMUNE RESPONSES DURING PREGNANCY AND LACTATION HPA AXIS AND IMMUNE RESPONSES}

Cytokines, peptide hormones, neurotransmitters, and their receptors are endogenous elements of the nervous, endocrine, and immune systems. These systems share ligands and receptors that serve to communicate (Haddad et al., 2002; Elenkov and

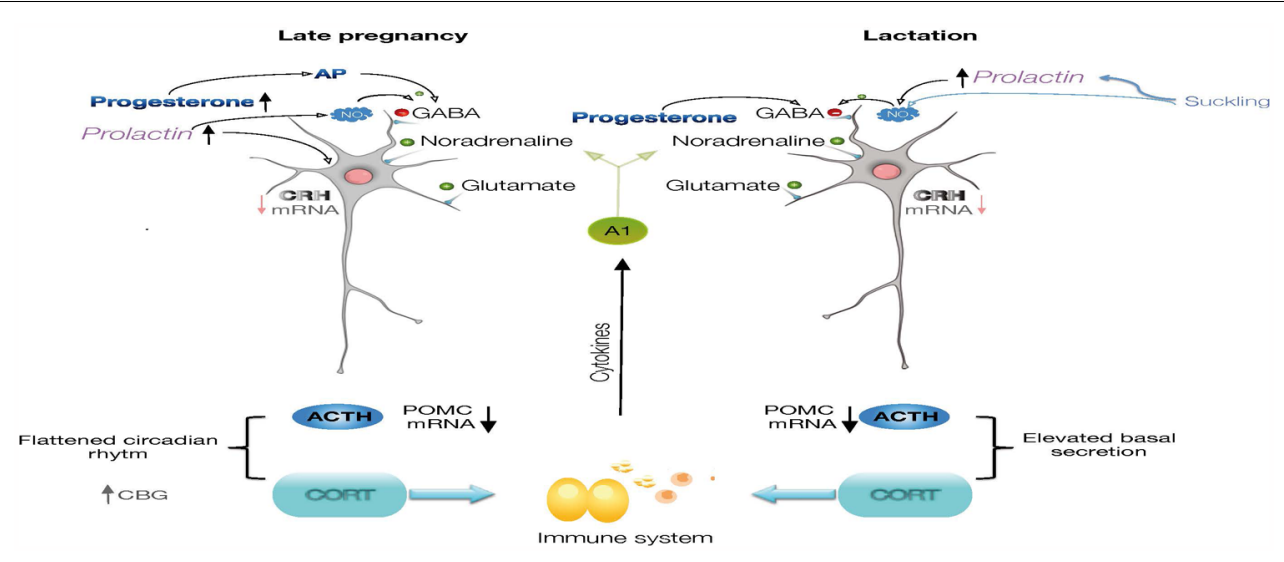

FIGURE 1 | Diagram illustrating the regulation of CRH expression in the parvocellular neurons of paraventricular nucleus of the hypothalamus. During late pregnancy and lactation, there is a diminished noradrenergic tone reaching $\mathrm{CRH}$ neurons, and progesterone, allopregnanolone, prolactin, and nitric oxide will contribute to the inhibitory regulation of the $\mathrm{CRH}$ neurons. The cascade of neuroendocrine responses, synthesis of $\mathrm{ACTH}$ and corticosteroids will also be modified. Neuroimmune interactions are represented by effects of glucocorticoids on cells of the immune system, and which molecules will affect CNS functions. $\mathrm{ACTH}$, adrenocorticotropic hormone; $\mathrm{AP}$, allopregnanolone; $\mathrm{CRH}$, corticotropin-releasing hormone; $\mathrm{CBG}$, corticosteroid-binding globulin; POMC, proopiomelanocortin. 
Chrousos, 2006). In addition to altered endocrine responses during lactation, stress also changes immune function in comparison to non-lactating animals (Monasterio and Morales, 2011), suggesting that bi-directional communication between the immune and endocrine systems is also altered during lactation. Pregnancy and lactation represent interesting experimental models that illustrate how the elevated basal plasma level of corticosterone and the diminished stress responses, affect the immune system. It is established that dampened HPA responses are associated with increased vulnerability to inflammation (Shanks et al., 1997, 1999) and therefore, alterations in endocrine regulation of immune responses during lactation may predispose the animal to inflammatory disease. However, not all of the immune responses are dampened during pregnancy and lactation (Jaedicke et al., 2009).

Several reports have documented motherhood-induced adaptations of the immune system in female rats, showing that this is a not always an immune suppression condition (Jaedicke et al., 2009). Early pregnancy has been associated with an increased cell density in the thymus and spleen, whereas late pregnancy and lactation have been associated with a decreased cellularity despite the expansion of the thymus medulla (Kendall and Clarke, 2000). Lactation delays the return of the thymus medulla to the original pre-pregnancy state. In addition, the changes in the thymus can be attributed to the adaptation of the maternal immune system to the semi-allogeneic fetus (Kendall and Clarke, 2000). Hormonal and neural changes can partially explain these modifications. Progesterone, PRL, and estradiol levels in plasma fluctuate in early, mid, and late pregnancy (Neville et al., 2002; Brusco et al., 2008). Nerve growth factor, nearly absent in the medulla of the adult thymus, is high in the medulla of the thymus during late pregnancy (Aloe et al., 1997). Also, lactation is associated with an increased susceptibility to parasitic infections (Barger, 1993), but much controversy exists in the effects of pregnancy and lactation on the progression of autoimmune diseases as documented by studies in rodents and in humans (Elenkov et al., 2001; Buchel et al., 2002; Vukusic et al., 2004; Gregg, 2009).

The immune system of lactating rodents has been the focus of only a few studies, which have shown that some immune functions become suppressed in this phase, while others remain unaffected or are enhanced (Jaedicke et al., 2009). For example, antibody production after immunization, and IL-6 (Monasterio and Morales, 2011) and IL-2 production in the spleen (Shanks et al., 1997) were suppressed during lactation in rodents. Conversely, evidence of increased concentrations of plasma IL-6 or an enhanced proliferative response of lymphocytes from mesenteric lymph nodes suggests activation of other immune responses (Shanks et al., 1997). In male rats, activation of the HPA axis and glucocorticoid release occurs during bacterial lipopolysaccharide (LPS) exposure, triggering an elaborate inflammatory response that involves the release of the pro-inflammatory mediators IL-1 $\beta$, IL- 6 , and tumor necrosis factor (TNF)- $\alpha$ (Grinevich et al., 2001), which stimulate cells to upregulate the inflammatory reaction, and systemically they activate the HPA axis (Chrousos, 1995). However, in lactating rats, LPS significantly elevates circulating levels of ACTH and corticosterone, but the magnitude of hormonal and hypothalamic responses to LPS are significantly reduced in lactating animals relative to virgin controls (Shanks et al., 1999). Despite this attenuated stress response, systemic immune responses to stressors are modulated during lactation indicating that the immune system is not generally suppressed but rather adjusted in this stage (Jaedicke et al., 2009).

In humans, the early postpartum period has been associated with up-regulated inflammatory responses and a relapse of autoimmune disorders such as rheumatoid arthritis and multiple sclerosis, often interpreted as a flare-up due to the rebound of the immune system after pregnancy (Elenkov et al., 2001; Buchel et al., 2002). However, long term-studies have shown that relapse rate of multiple sclerosis remains similar to pre-pregnancy level, after an increase in the first trimester postpartum (Vukusic et al., 2004). A broad state of immune activation is also characteristic of the early postpartum period, as measured by levels of neopterin, soluble IL-2 receptor, and soluble CD8 antigen (Burns et al., 1999). This may help women recover from the biological stress of parturition, but more studies about the magnitude and length of such a state are necessary. Immune and inflammatory activation in postpartum women may be factors leading to anxiety and depression in the early days after delivery (Maes et al., 2000). CD4 cell counts are reported to rise during postpartum, primarily due to $\gamma \delta \mathrm{T}$ cells (Watanabe et al., 1996). Natural killer (NK) subsets with weak cytotoxic activity (CD16+, CD57+) were found to increase during months 1-4 postpartum (Watanabe et al., 1997), and lymphocyte proliferation was higher than non-postpartum controls. Furthermore, the postpartum period is also associated with the onset of autoimmune thyroid syndrome (Muller et al., 2001). Thus, immune responses linked to reproduction can either be dampened or enhanced depending on the stimulus and hormonal status (De Bellis et al., 2005; Hughes, 2012; Shelly et al., 2012).

In summary, during both pregnancy and lactation the immune responses are altered, but instead of considering them to be immune-suppressed stages, they represent reproductive conditions in which either stress or immune responses can vary depending on the hormonal status (Mor and Cardenas, 2010). The immunology of reproduction is the result of the combination of signals and responses originating from the fetal-placental and the maternal immune systems. This last is under the regulation of the CNS through the hormonal (glucocorticoids) stress response, pituitary responses, and the autonomic nervous system.

\section{PROGESTERONE ACTIONS ON CNS IMMUNE RESPONSES}

Among the hormones that have been considered candidate inducers of pregnancy- and lactation-related adaptations in HPA axis and immune responses is progesterone. Progesterone is a steroid hormone synthesized by the corpus luteum in cycling females and by the placenta during pregnancy; it can enter the brain from the circulation and can also be synthesized in the brain by oligodendrocytes and excitatory neurons (Stein, 2011). During pregnancy, progesterone levels in plasma and brain are increased and are accompanied by elevated levels of its metabolite allopregnanolone (Brunton and Russell, 2008; Mostallino et al., 2009).

Progesterone (and its metabolite allopregnanolone) has important actions in the female's brain, such as the expression of 
maternal behavior (Bridges, 1984). During pregnancy, high levels of allopregnanolone suppress HPA -axis responses to stress: allopregnanolone may enhance the action of GABA in the PVN or on afferent inputs to the CRH neurons to suppress stress responses, and it also induces and maintains the endogenous inhibitory opioid mechanism in the nucleus of the solitary tract (Brunton et al., 2009). Blocking allopregnanolone restores HPA axis responses to systemically administered IL- $1 \beta$ in late pregnant rats (Brunton and Russell, 2008). Allopregnanolone acts as an agonist on the GABA receptor, exerting anxiolytic, sedative, and antiepileptic effects, and it enhances the myelination/remyelination process in the central and peripheral nervous system (Wang et al., 2008; Gangisetty and Reddy, 2010). These steroid hormones have been described as potent regulators of growth factor expression during pregnancy: epidermal growth factor (EGF), insulin-like growth factor (IGF), and transforming growth factor (TGF- $\beta 1$ ) in particular are all up-regulated, promoting neural proliferation (Wang et al., 2008). Moreover, they increase neurogenesis within the subgranular zone of the dentate gyrus and subventricular zone (Pawluski etal., 2009), and induce regenerative responses in a mouse model of Alzheimer's disease (Pike et al., 2009; Borowicz et al., 2011). During lactation, progesterone participates in glial changes in brain areas such as the cingulate cortex (Salmaso et al., 2009) and the dentate gyrus of the hippocampus (Cabrera et al., 2013), and this steroid is part of the hormonal cocktail responsible for diminished responses of astrocyte and microglial cells in the hippocampus of lactating rats to damage induced by excitotoxic insults (Cabrera et al., 2013).

Progesterone and allopregnanolone attenuate traumatic brain injury, and diminish the elevation of pro-inflammatory cytokines in a time-dependent manner, suggesting that the protection occurs by limiting the overexpression of cytokines, when they peak at $3 \mathrm{~h}$ after a brain injury, rather than inhibiting their expression later in the post-injury cascade of toxic events (He et al., 2004). Both steroids prevent breakdown of the blood-brain barrier in edema and stroke (Ishrat et al., 2010). Progesterone exerts some of its actions through the intracellular, membrane-bound progesterone receptor, while allopregnanolone does not bind to the progesterone receptor (Ishrat et al., 2010). Also, progesterone can reduce the excessive excitotoxicity and inflammation by stimulating activation of the neuroprotective mitogen-activated protein kinase (MAPK) and PI3-K pathways (Kaur et al., 2007). Through use of calcium imaging, electrophysiology, and the measurement of changes in activity-dependent gene expression, progesterone was found to block calcium entry through voltage-gated calcium channels, leading to alterations in the signaling of the activitydependent transcription factors nuclear factor of activated T-cells (NFAT) and cAMP response element-binding protein (CREB; Luoma et al., 2012). This effect of progesterone on calcium signaling provides a putative mechanism for its neuroprotective actions (Luoma et al., 2012). Furthermore, allopregnanolone is an allosteric modifier of the GABAA receptors expressed by oxytocin neurons (Koksma et al., 2003). Electrophysiological studies of hypothalamic oxytocin neurons showed that allopregnanolone acts via a $\mathrm{G}$-protein mechanism involving protein kinase $\mathrm{C}$ to delay the closure of the $\mathrm{Cl}^{-}$channel after activation (Brussaard and Koksma, 2003), enhancing both the tonic and phasic actions of GABA in oxytocin neurons. This modulatory action on GABA transmission represents an alternative candidate pathway for the protective actions of this steroid.

Progesterone also plays an important role in the periphery. Progesterone-dependent immunomodulation is one of the mechanisms that enable pregnancy to proceed to term, because it protects the fetus from immunological rejection. Recent evidence suggests that autocrine/paracrine factors such as cytokines play a crucial role, possible as effectors of steroid hormones (Agrawal et al., 2011). A growing body of evidence implicates progesterone in the establishment of an adequate immune response during pregnancy (Hirsch and Muhle, 2002; Aisemberg et al., 2013).

Thus, progesterone and its metabolite allopregnanolone are important modulators of the immune responses in the periphery and within the CNS. Such effects are aimed to diminish the impact of an acute lesion or degenerative process in the nervous system while in the periphery contributes to the regulation of immune response against the fetus (Aisemberg et al., 2013).

\section{PROLACTIN ACTIONS ON CNS AND IMMUNE SYSTEM}

Prolactin is a peptide hormone secreted from the anterior pituitary into the circulation; it is thought to cross the blood-brain barrier and is known to regulate a wide variety of physiological process (Bole-feysot et al., 1998; Grattan and Kokay, 2008). PRL is also produced in a broad spectrum of extrapituitary sites including cells of the nervous and the immune system (Montgomery, 2001; Torner etal., 2002; Ignacak etal., 2012), and it is an important mediator of the immunoneuroendocrine network. However, effects of PRL on the immune system are complex. Removal of PRL by hypophysectomy impairs thymus growth (Nagy and Berczi, 1978) and immune reaction to immunogenic factors (Bernton et al., 1988) in rats. On the other hand, hyperprolactinemia (HP), in mice injected with Listeria monocytogenes increases mortality associated with impaired lymphocyte proliferation and decreased macrophage-activating factor production by T lymphocytes (Bernton et al., 1988). Furthermore, a PRL-like mRNA and a secreted product have been detected in human B-lymphoblastoid cell lines (Bernton et al., 1988; Baglia et al., 1991). Peripheral blood mononuclear cells also secrete a PRL-like protein, suggesting that it binds to PRL receptors and migrates to the nucleus, where it serves as a co-mitogen and autocrine regulator of cell growth. But, there are several reports showing that PRL is not essential for the proper development and function of the mouse immune system. Using PRL-deficient animals it was shown that PRL is not required for normal hematopoiesis (Horseman et al., 1997), and that PRL receptor signaling is not required for normal immunity (Bouchard et al., 1999). PRL is known to have other contradictory actions on the immune system that depends upon the concentration: it inhibits lymphocyte proliferation at high concentrations, while having enhancing effects at lower concentrations (Matera et al., 1992).

During pregnancy in rats, PRL plasma levels are high in the first half, they decrease until term, and then rise again at postpartum and throughout lactation (Neville et al., 2002). PRL actions in early gestation are crucial to prepare the mammary gland for lactation and in the CNS to establish the appropriate adaptive behavioral responses of the mother (Grattan and Kokay, 2008) 
toward the pups. PRL has been related to behavioral and neuronal effects, such as maternal behavior (Mann and Bridges, 2001), attenuation of anxiety and hormonal and neuronal responses to various stressors (Lightman et al., 2001; Torner and Neumann, 2002; Donner etal., 2007), neurogenesis (Shingo etal., 2003; Torner et al., 2009; Larsen and Grattan, 2010; Walker et al., 2012), and neuroprotection (Torner et al., 2009; Tejadilla et al., 2010).

Inflammatory response after a brain injury, such as proliferation and activation of glia is enhanced by PRL (Möderscheim et al., 2007), and elevated levels of PRL stimulate mitogenesis in astrocyte and oligodendrocyte populations of the subventricular zone (Larsen and Grattan, 2010). PRL also increases oligodendrocyte precursor cell proliferation, which in turn enhances the capacity to generate new oligodendrocytes and myelination. This process is associated with the capacity to repair white matter damage in the maternal CNS by increasing myelin basic protein expression (Gregg, 2009). During lactation, a physiological hyperprolactinemic state, there is a reduced sensitivity to kainic acid-induced cell damage in the dorsal hippocampus of the dam, showing that lactation is a natural model of neuroprotection (Vanoye-Carlo et al., 2008; Cabrera et al., 2009) in which PRL can participate. PRL systemic administration has been reported to protect the dorsal hippocampus of female rats against excitotoxicity induced by kainic acid administration, blocking cell loss and neurodegeneration, and diminishing the progression of kainategenerated behavioral manifestation of seizures (Tejadilla et al., 2010). Overall, these studies on protective effects of PRL within the CNS draw attention to the importance of studying females, and the relevance of neuroendocrine-immune interactions when investigating effects of this hormone.

An interesting example of the neuroendocrine-immune interactions is seen in the HP occurring in patients with autoimmune diseases, such as rheumatoid arthritis and systemic lupus erythematosus, and with organ-specific autoimmune diseases, as celiac disease, type 1 diabetes mellitus, Addison's disease, autoimmune thyroid diseases (reviewed by, De Bellis et al., 2005). In these diseases PRL increases the synthesis of IFN $\gamma$ and IL-2 by Th1 lymphocytes. Moreover, PRL activates Th2 lymphocytes with autoantibody production. The inhibitory effects of IL-1ß on the tuberoinfundibular dopaminergic neurons that inhibit PRL secretion could explain this HP. González et al. (2004), showed that i.c.v. injection of LPS in rats, produces a decrease in tyrosine hydroxylase $(\mathrm{TH})$ activity in the medial eminence, an increase in the serum levels of PRL, and a decrease in the number of TH- and TH mRNA-positive cells in the arcuate nucleus, indicating that dopamine neurons of the hypothalamus are functionally susceptible to local inflammatory stimuli. Additionally, treatment with the dopamine-agonist, bromocriptine, inhibits both PRL secretion and the severity of acute experimental encephalomyelitis (Riskind et al., 1991). This is an example of how molecules of the immune system could affect the neurons in the hypothalamus increasing the secretion of PRL, which in its turn will enhance peripheral inflammatory responses.

\section{CONCLUSION}

In summary, during pregnancy and lactation, responses of the HPA axis and the immune system are altered and clearly regulated by suckling and hormone fluctuation. Pregnancy and lactation are the most important periods for the conservation of the species, and they represent fundamental stages at which both mother and offspring must be protected. The immune system is crucial to protect the mother and the product against the environment and there is evidence supporting the notion that immunity is suppressed during motherhood. In this sense, the immune responses of the mother should be adjusted to conserve defenses, but should be tuned to preserve the developing offspring (Mor and Cardenas, 2010). Therefore, pregnancy and lactation are unique conditions in which the immune system is modulated or adjusted, but not fully suppressed.

\section{AUTHOR CONTRIBUTIONS}

Nela Monasterio conceived the main premises that are the focus of this review and wrote the largest portion of the paper; Edgar Vergara wrote important subsections of the paper; and Teresa Morales conceived the main premises that are the focus of this review, designed the structure of the paper, wrote a large portion of the paper, and edited the final version.

\section{ACKNOWLEDGMENTS}

The authors thank Dr. Dorothy Pless for grammatical review. Cited work from our group was supported by PAPIIT-DGAPA, UNAM, and CONACYT, México. We are grateful to the reviewers whose comments help to improve this work.

\section{REFERENCES}

Agrawal, V., Jaiswal, M. K., and Jaiswal, Y. K. (2011). Lipopolysaccharide induces alterations in ovaries and serum level of progesterone and $17 \beta$-estradiol in the mouse. Fertil. Steril. 95, 1471-1474. doi: 10.1016/j.fertnstert.2010.08.046

Aisemberg, J., Vercelli, C. A., Bariani, M. V., Billi, S. C., Wolfson, M. L., and Franchi, A. M. (2013). Progesterone is essential for protecting against LPSinduced pregnancy loss. LIF as a potential mediator of the anti-inflammatory effect of progesterone. PLoS ONE 8:e56161. doi: 10.1371/ journal.pone.0056161

Aloe, L., Bracci-Laudiero, L., Bonini, S., and Manni, L. (1997). The expanding role of nerve growth factor: from neurotrophic activity to immunologic diseases. Allergy 52, 883-894. doi: 10.1111/j.1398-9995.1997.tb01247.x

Atkinson, H. C., and Waddell, B. J. (1995). The hypothalamic-pituitary-adrenal axis in rat pregnancy and lactation: circadian variation and interrelationship of plasma adrenocorticotropin and corticosterone. Endocrinology 136, 512-520. doi: 10.1210/en.136.2.512

Baglia, L. A., Cruz, D., and Shaw, J. E. (1991). An Epstein-Barr virus-negative Burkitt lymphoma cell line (sfRamos) secretes a prolactin-like protein during continuous growth in serum-free medium. Endocrinology 128, 2266-2272. doi: 10.1210/endo-128-5-2266

Barger, I. A. (1993). Influence of sex and reproductive status on susceptibility of ruminants to nematode parasitism. Int. J. Parasitol. 23, 463-469. doi: 10.1016/0020-7519(93)90034-V

Bernton, E. W., Meltzer, M. S., and Holaday, J. W. (1988). Suppression of macrophage activation and T-lymphocyte function in hypoprolactinemic mice. Science 239, 401-404. doi: 10.1126/science.3122324

Bole-feysot, C., Goffin, V., Edery, M., Binart, N., and Kelly, P. A. (1998). Prolactin (PRL) and its receptor: actions, signal transduction pathways and phenotypes observed in PRL receptor knockout mice. Endocr. Rev. 19, 225-268. doi: 10.1210/er.19.3.225

Borowicz, K. K., Piskorska, B., Banach, M., and Czuczwar, S. J. (2011). Neuroprotective actions of neurosteroids. Front. Endocrinol. 2:50. doi: 10.3389/fendo.2011.00050

Bouchard, B., Ormandy, C. J., Di Santo, J. P., and Kelly, P. A. (1999). Immune system development and function in prolactin receptor-deficient mice. J. Immunol. 163, 576-582. 
Bridges, R. S. (1984). A quantitative analysis of the roles of dosage, sequence, and duration of estradiol and progesterone exposure in the regulation of maternal behavior in the rat. Endocrinology 114, 930-940. doi: 10.1210/endo-114-3-930

Brunton, P. J., McKay, A. J., Ochedalski, T., Piastowska, A., Rebas, E., Lachowicz, A., et al. (2009). Central opioid inhibition of neuroendocrine stress responses in pregnancy in the rat is induced by the neurosteroid allopregnanolone. J. Neurosci. 29, 6449-6460. doi: 10.1523/JNEUROSCI.0708-09.2009

Brunton, P. J., Meddle, S. L., Ma, S., Ochedalski, T., Douglas, A. J., and Russell, J. A. (2005). Endogenous opioids and attenuated hypothalamic-pituitary-adrenal axis responses to immune challenge in pregnant rats. J. Neurosci. 25, 5117-5126. doi: 10.1523/JNEUROSCI.0866-05.2005

Brunton, P. J., and Russell, J. A. (2008). Attenuated hypothalamo-pituitary-adrenal axis responses to immune challenge during pregnancy: the neurosteroid opioid connection. J. Physiol. 586, 369-375. doi: 10.1113/jphysiol.2007.146233

Brunton, P. J., and Russell, J. A. (2011). Allopregnanolone and suppressed hypothalamo-pituitary-adrenal axis stress responses in late pregnancy in the rat. Stress 14, 6-12. doi: 10.3109/10253890.2010.482628

Brusco, J., Wittmann, R., de Azevedo, M. S., Lucion, A. B., Franci, C. R., Giovenardi, M., et al. (2008). Plasma hormonal profiles and dendritic spine density and morphology in the hippocampal CAl stratum radiatum, evidenced by ligh microscopy, of virgin and postpartum female rats. Neurosci. Lett. 438, 346-350. doi: 10.1016/j.neulet.2008.04.063

Brussaard, A. B., and Koksma, J.-J. (2003). Conditional regulation of neurosteroid sensitivity of GABAA receptors. Ann. N. Y. Acad. Sci. 1007, 29-36. doi: 10.1196/annals. 1286.003

Buchel, E., Van Steenbergen, W., Nevens, F., and Fevery, J. (2002). Improvement of autoimmune hepatitis during pregnancy followed by flare-up after delivery. Am. J. Gastroenterol. 97, 3160-3165. doi: 10.1111/j.1572-0241.2002.07124.x

Burns, D. N., Nourjah, P., Wright, D. J., Minkoff, H., Landesman, S., Rubinstein, A., et al. (1999). Changes in immune activation markers during pregnancy and postpartum. J. Reprod. Immunol. 42, 147-165. doi: 10.1016/S0165-0378(98)00085-0

Cabrera, V., Cantú, D., Ramos, E., Vanoye-Carlo, A., Cerbón, M., and Morales, T. (2009). Lactation is a natural model of hippocampus neuroprotection against excitotoxicity. Neurosci. Lett. 461, 136-139. doi: 10.1016/j.neulet.2009.06.017

Cabrera, V., Ramos, E., González-Arenas, A., Cerbón, M., Camacho, I., and Morales, T. (2013). Lactation reduces glial activation induced by excitotoxicity in the rat hippocampus. J. Neuroendocrinol. 25, 519-527. doi: 10.1111/jne.12028

Chrousos, G. P. (1995). The hypothalamic-pituitary-adrenal axis and immune-mediated inflammation. N. Engl. J. Med. 332, 1351-1362. doi: 10.1056/NEJM199505183322008

da Costa, A. P., Ma, X., Ingram, C. D., Lightman, S. L., and Aguilera, G. (2001). Hypothalamic and amygdaloid corticotropin-releasing hormone (CRH) and CRH receptor-1 mRNA expression in the stress-hyporesponsive late pregnant and early lactating rat. Brain Res. Mol. Brain Res. 91, 119-130. doi: 10.1016/S0169-328X(01)00137-1

da Costa, A. P., Wood, S., Ingram, C. D., and Lightman, S. L. (1996). Region-specific reduction in stress-induced c-fos mRNA expression during pregnancy and lactation. Brain Res. 742, 177-184. doi: 10.1016/S0006-8993(96) 00962-6

De Bellis, A., Bizzarro, A., Pivonello, R., Lombardi, G., and Bellastella, A. (2005) Prolactin and autoimmunity. Pituitary 8, 25-30. doi: 10.1007/s11102-005-5082-5

Donner, N., Bredewold, R., Maloumby, R., and Neumann, I. D. (2007). Chronic intracerebral prolactin attenuates neuronal stress circuitries in virgin rats. Eur. J. Neurosci. 25, 1804-1814. doi: 10.1111/j.1460-9568.2007.05416.x

Douglas, A. J., Johnstone, H., Brunton, P., and Russell, J. A. (2000). Sex-steroid induction of endogenous opioid inhibition on oxytocin secretory responses to stress. J. Neuroendocrinol. 12, 343-350. doi: 10.1046/j.1365-2826.2000.00460.x

Douglas, A. J., Meddle, S. L., Toschi, N., Bosch, O. J., and Neumann, I. D. (2005). Reduced activity of the noradrenergic system in the paraventricular nucleus at the end of pregnancy: implications for stress hyporesponsiveness. J. Neuroendocrinol. 17, 40-48. doi: 10.1111/j.1365-2826.2005.01272.x

Elenkov, I. J., and Chrousos, G. P. (2006). Stress system-organization, physiology and immunoregulation. Neuroimmunomodulation 13, 257-267. doi: $10.1159 / 000104853$

Elenkov, I. J., Wilder, R. L., Bakalov, V. K., Link, A. A., Dimitrov, M. A., Fisher, S., et al. (2001). IL-12, TNF-alpha, and hormonal changes during late pregnancy and early postpartum: implications for autoimmune disease activity during these times. J. Clin. Endocrinol. Metab. 86, 4933-4938. doi: 10.1210/jc.86.10.4933
Fischer, D., Patchev, V. K., Hellbach, S., Hassan, A. H., and Almeida, O. F. (1995). Lactation as a model for naturally reversible hypercorticalism plasticity in the mechanisms governing hypothalamo-pituitary- adrenocortical activity in rats. J. Clin. Invest. 96, 1208-1215. doi: 10.1172/JCI118153

Gangisetty, O., and Reddy, D. S. (2010). Neurosteroid withdrawal regulates GABA-A receptor $\alpha 4$-subunit expression and seizure susceptibility by activation of progesterone receptor-independent early growth response factor-3 pathway. Neuroscience 170, 865-880. doi: 10.1016/j.neuroscience.2010.07.037

González, M. C., Abreu, P., Barroso-Chinea, P., Cruz-Muros, I., and GonzálezHernández, T. (2004). Effect of intracerebroventricular injection of lipopolysaccharide on the tuberoinfundibular dopaminergic system of the rat. Neuroscience 127, 251-259. doi: 10.1016/j.neuroscience.2004.04.057

Grattan, D. R., and Kokay, I. C. (2008). Prolactin: a pleiotropic neuroendocrine hormone. J. Neuroendocrinol. 20, 752-763. doi: 10.1111/j.1365-2826.2008.01736.x

Gregg, C. (2009). Pregnancy, prolactin and white matter regeneration. J. Neurol. Sci. 285, 22-27. doi: 10.1016/j.jns.2009.06.040

Grinevich, V., Ma, X. M., Herman, J. P., Jezova, D., Akmayev, I., and Aguilera, G. (2001). Effect of repeated lipopolysaccharide administration on tissue cytokine expression and hypothalamic-pituitary-adrenal axis activity in rats. J. Neuroendocrinol. 13, 711-723. doi: 10.1046/j.1365-2826.2001.00684.x

Haddad, J. J., Saadé, N. E., and Safieh-Garabedian, B. (2002). Cytokines and neuro-immune-endocrine interactions: a role for the hypothalamic-pituitaryadrenal revolving axis. J. Neuroimmunol. 133, 1-19. doi: 10.1016/S0165-5728(02) 00357-0

He, J., Evans, C.-O., Hoffman, S. W., Oyesiku, N. M., and Stein, D. G. (2004). Progesterone and allopregnanolone reduce inflammatory cytokines after traumatic brain injury. Exp. Neurol. 189, 404-412. doi: 10.1016/j.expneurol.2004.06.008

Hirsch, E., and Muhle, R. (2002). Intrauterine bacterial inoculation induces labor in the mouse by mechanisms other than progesterone withdrawal. Biol. Reprod. 67, 1337-1341. doi: 10.1095/biolreprod67.4.1337

Hoffman, G. E., Le, W. W., Abbud, R., Lee, W. S., and Smith, M. S. (1994). Use of Fos-related antigens (FRAs) as markers of neuronal activity: FRA changes in dopamine neurons during proestrus, pregnancy and lactation. Brain Res. 654, 207-215. doi: 10.1016/0006-8993(94)90481-2

Horseman, N. D., Zhao, W., Montecino-Rodriguez, E., Tanaka, M., Nakashima, K., Engle, S. J., et al. (1997). Defective mammopoiesis, but normal hematopoiesis, in mice with a targeted disruption of the prolactin gene. EMBO J. 16, 6926-6935. doi: 10.1093/emboj/16.23.6926

Hughes, G. C. (2012). Progesterone and autoimmune disease. Autoimmun. Rev. 11, A502-A514. doi: 10.1016/j.autrev.2011.12.003

Ignacak, A., Kasztelnik, M., and Sliwa, T. (2012). Prolactin-not only lactotrophin. A "new" view of the "old" hormone. J. Physiol. Pharmacol. 63, 435-443.

Ishrat, T., Sayeed, I., Atif, F., Hua, F., and Stein, D. G. (2010). Progesterone and allopregnanolone attenuate blood-brain barrier dysfunction following permanent focal ischemia by regulating the expression of matrix metalloproteinases. Exp. Neurol. 226, 183-190. doi: 10.1016/j.expneurol.2010.08.023

Jaedicke, K. M., Fuhrmann, M. D., and Stefanski, V. (2009). Lactation modifies stress-induced immune changes in laboratory rats. Brain. Behav. Immun. 23, 700-708. doi: 10.1016/j.bbi.2009.02.005

Johnstone, H. A., Wigger, A., Douglas, A. J., Neumann, I. D., Landgraf, R., Seckl, J. R., et al. (2000). Attenuation of hypothalamic-pituitary-adrenal axis stress responses in late pregnancy: changes in feedforward and feedback mechanisms. J. Neuroendocrinol. 12, 811-822. doi: 10.1046/j.1365-2826.2000.00525.x

Kaur, P., Jodhka, P. K., Underwood, W. A., Bowles, C. A., de Fiebre, N. C., de Fiebre, C. M. et al. (2007). Progesterone increases brain-derived neuroptrophic factor expression and protects against glutamate toxicity in a mitogen-activated protein kinase- and phosphoinositide-3 kinase-dependent manner in cerebral cortical explants. J. Neurosci. Res. 85, 2441-2449. doi: 10.1002/jnr.21370

Kendall, M. D., and Clarke, A. G. (2000). The thymus in the mouse changes its activity during pregnancy: a study of the microenvironment. J. Anat. 197(Pt 3), 393-411. doi: 10.1046/j.1469-7580.2000.19730393.x

Kinsley, C. H., and Lambert, K. G. (2008). Reproduction-induced neuroplasticity: natural behavioural and neuronal alterations associated with the production and care of offspring. J. Neuroendocrinol. 20, 515-525. doi: 10.1111/j.13652826.2008.01667

Koksma, J.-J., van Kesteren, R. E., Rosahl, T. W., Zwart, R., Smit, A. B., Lüddens, H., et al. (2003). Oxytocin regulates neurosteroid modulation of GABA(A) receptors in supraoptic nucleus around parturition. J. Neurosci. 23, 788-797. 
Larsen, C. M., and Grattan, D. R. (2010). Prolactin-induced mitogenesis in the subventricular zone of the maternal brain during early pregnancy is essential for normal postpartum behavioral responses in the mother. Endocrinology 151, 3805-3814. doi: 10.1210/en.20091385

Lightman, S. L., Windle, R., Da Costa, A., Shanks, N., and Ingram, C. (1998). “Lactation: a physiological model of stress hyporesponsiveness of the neuroendocrine system," in New Frontiers in Stress Research. Modulation of Brain Function, ed A. Levy, E. Grauer, D. Ben-Nathan, and E. R. de Kloet (Switzerland: Harwood Academic Publishers GmbH), 59-71.

Lightman, S. L., Windle, R. J., Wood, S. A., Kershaw, Y. M., Shanks, N., and Ingram, C. D. (2001). Peripartum plasticity within the hypothalamo-pituitaryadrenal axis. Prog. Brain Res. 133, 111-129. doi: 10.1016/S0079-6123(01) 33009-1

Lightman, S. L., and Young, W. S. III. (1989). Lactation inhibits stress-mediated secretion of corticosterone and oxytocin and hypothalamic accumulation of corticotropin-releasing factor and enkephalin messenger ribonucleic acids. Endocrinology 124, 2358-2364. doi: 10.1210/endo-124-5-2358

Luoma, J. I., Stern, C. M., and Mermelstein, P. G. (2012). Progesterone inhibition of neuronal calcium signaling underlies aspects of progesteronemediated neuroprotection. J. Steroid Biochem. Mol. Biol. 131, 30-36. doi: 10.1016/j.jsbmb.2011.11.002

Ma, S., Shipston, M. J., Morilak, D., and Russell, J. A. (2005). Reduced hypothalamic vasopressin secretion underlies attenuated adrenocorticotropin stress responses in pregnant rats. Endocrinology 146, 1626-1637. doi: 10.1210/en.2004-1368

Maes, M., Lin, A. H., Ombelet, W., Stevens, K., Kenis, G., De Jongh, R., et al. (2000). Immune activation in the early puerperium is related to postpartum anxiety and depressive symptoms. Psychoneuroendocrinology 25, 121-137. doi: 10.1016/S0306-4530(99)00043-8

Mann, P. E., and Bridges, R. S. (2001). Lactogenic hormone regulation of maternal behavior. Prog. Brain Res. 133, 251-262. doi: 10.1016/S0079-6123(01) 33019-4

Matera, L., Cesano, A., Bellone, G., and Oberholtzer, E. (1992). Modulatory effect of prolactin on the resting and mitogen-induced activity of T, B, and NK lymphocytes. Brain Behav. Immun. 6, 409-417. doi: 10.1016/0889-1591(92)90039-Q

Möderscheim, T. A. E., Gorba, T., Pathipati, P., Kokay, I. C., Grattan, D. R., Williams, C. E., et al. (2007). Prolactin is involved in glial responses following a focal injury to the juvenile rat brain. Neuroscience 145, 963-973. doi: 10.1016/j.neuroscience.2006.12.053

Monasterio, N., and Morales, T. (2011). Nitric oxide has a role in attenuating the neuroendocrine response to anaphylactoid stress during lactation. Brain Res. 1402, 54-66. doi: 10.1016/j.brainres.2011.05.062

Montgomery, D. W. (2001). Prolactin production by immune cells. Lupus 10, 665 675. doi: 10.1191/096120301717164895

Mor, G., and Cardenas, I. (2010). The immune system in pregnancy: a unique complexity. Am. J. Reprod. Immunol. 63, 425-433. doi: 10.1111/j.16000897.2010.00836.x

Morales, T. (2011). Recent findings on neuroprotection against excitotoxicity in the hippocampus of female rats. J. Neuroendocrinol. 23, 994-1001. doi: 10.1111/j.1365-2826.2011.02141.x

Mostallino, M. C., Sanna, E., Concas, A., Biggio, G., and Follesa, P. (2009) Plasticity and function of extrasynaptic GABA(A) receptors during pregnancy and after delivery. Psychoneuroendocrinology 34(Suppl. 1), S74-S83. doi: 10.1016/j.psyneuen.2009.06.013

Muller, A. F., Drexhage, H. A., and Berghout, A. (2001). Postpartum thyroiditis and autoimmune thyroiditis in women of childbearing age: recent insights and consequences for antenatal and postnatal care. Endocr. Rev. 22, 605-630. doi: 10.1210/er.22.5.605

Nagy, E., and Berczi, I. (1978). Immunodeficiency in hypophysectomized rats. Acto Endocrinol. 89, 530-537. doi: 10.1530/acta.0.0890530

Neumann, I. D., Bosch, O. J., Toschi, N., Torner, L., and Douglas, A. J. (2003). No stress response of the hypothalamo-pituitary-adrenal axis in parturient rats: lack of involvement of brain oxytocin. Endocrinology 144, 2473-2479. doi: 10.1210/en.2003-0037

Neumann, I. D., Johnstone, H. A., Hatzinger, M., Liebsch, G., Shipston, M., Russell, J. A., et al. (1998). Attenuated neuroendocrine responses to emotional and physical stressors in pregnant rats involve adenohypophysia changes. J. Physiol. 508(Pt 1), 289-300. doi: 10.1111/j.1469-7793.1998. 289br.x
Neville, M. C., McFadden, T. B., and Forsyth, I. (2002). Hormonal regulation of mammary differentiation and milk secretion. J. Mammary Gland Biol. Neoplasia 7, 49-66. doi: 10.1023/A:1015770423167

Otukonyong, E. E., Okere, C. O., Johnstone, L. E., Murata, T., Kaba, H., and Higuchi, T. (2000). Effect of suckling on NADPH-diaphorase (Nitric oxide synthase, NOS) reactivity and NOS gene expression in the paraventricular and supraoptic nuclei of lactating rats. J. Neuroendocrinol. 12, 1001-1008. doi: 10.1046/j.1365-2826.2000.00548.x

Parker, V. J., Menzies, J. R., and Douglas, A. J. (2011). Differential changes in the hypothalamic-pituitary-adrenal axis and prolactin responses to stress in early pregnant mice. J. Neuroendocrinol. 23, 1066-1078. doi: 10.1111/j.13652826.2011.02204.x

Patel, H., Chowdrey, H. S., Jessop, D. S., and Lightman, S. L. (1993) Hormonal responses to central alpha-1 receptor stimulation during lactation. Ann. N. Y. Acad. Sci. 689, 489-491. doi: 10.1111/j.1749-6632.1993.tb 55577.x

Pawluski, J. L., Brummelte, S., Barha, C. K., Crozier, T. M., and Galea, L. A. (2009). Effects of steroid hormones on neurogenesis in the hippocampus of the adult female rodent during the estrous cycle, pregnancy, lactation and aging. Front. Neuroendocrinol. 30:343-357. doi: 10.1016/j.yfrne.2009. 03.007

Pike, C. J., Carroll, J. C., Rosario, E. R., and Barron, A. M. (2009). Protective actions of sex steroid hormones in Alzheimer's disease. Front. Neuroendocrinol. 30:239-258. doi: 10.1016/j.yfrne.2009.04.015

Popeski, N., Amir, S., and Woodside, B. (1999). Changes in NADPH-d staining in the paraventricular and supraoptic nuclei during pregnancy and lactation in rats: role of ovarian steroids and oxytocin. J. Neuroendocrinol. 11, 53-61. doi: 10.1046/j.1365-2826.1999.00291.x

Riskind, P. N., Massacesi, L., Doolittle, T. H., and Hauser, S. L. (1991). The role of prolactin in autoimmune demyelination: suppression of experimental allergic encephalomyelitis by bromocriptine. Ann. Neurol. 29, 542-547. doi: 10.1002/ana.410290514

Russell, J. A., Douglas, A. J., and Brunton, P. J. (2008). Reduced hypothalamopituitary-adrenal axis stress responses in late pregnancy: central opioid inhibition and noradrenergic mechanisms. Ann. N. Y. Acad. Sci. 1148, 428-438. doi: 10.1196/annals.1410.032

Salmaso, N., Nadeau, J., and Woodside, B. (2009). Steroid hormones and maternal experience interact to induce glial plasticity in the cingulate cortex. Eur. $J$. Neurosci. 29, 786-794. doi: 10.1111/j.1460-9568.2009.06627.x

Serafim, A. P., and Felicio, L. F. (2002). Reproductive experience influences grooming behavior during pregnancy in rats. Braz. J. Med. Biol. Res. 35, 391-394. doi: 10.1590/S0100-879X2002000300016

Shanks, N., Kusnecov, A., Pezzone, M., Berkun, J., and Rabin, B. S. (1997). Lactation alters the effects of conditioned stress on immune function. Am. J. Physiol. 272, R16-R25.

Shanks, N., Windle, R. J., Perks, P., Wood, S., Ingram, C. D., and Lightman, S. L. (1999). The hypothalamic-pituitary-adrenal axis response to endotoxin is attenuated during lactation. J. Neuroendocrinol. 11, 857-865. doi: 10.1046/j.1365-2826.1999.00400.x

Shelly, S., Boaz, M., and Orbach, H. (2012). Prolactin and autoimmunity. Autoimmun. Rev. 11, A465-A470. doi: 10.1016/j.autrev.2011.11.009

Shingo, T., Gregg, C., Enwere, E., Fujikawa, H., Hassam, R., Geary, C. etal. (2003). Pregnancy-stimulated neurogenesis in the adult female forebrain mediated by prolactin. Science 299, 117-120. doi: 10.1126/science. 1076647

Stein, D. G. (2011). Progesterone in the treatment of acute traumatic brain injury: a clinical perspective and update. Neuroscience 191, 101-106. doi: 10.1016/j.neuroscience.2011.04.013

Stern, J. M., Goldman, L., and Levine, S. (1973). Pituitary-adrenal responsiveness during lactation in rats. Neuroendocrinology 12, 179-191. doi: 10.1159/000122167

Tejadilla, D., Cerbón, M., and Morales, T. (2010). Prolactin reduces the damaging effects of excitotoxicity in the dorsal hippocampus of the female rat independently of ovarian hormones. Neuroscience 169, 1178-1185. doi: 10.1016/j.neuroscience.2010.05.074

Tilbrook, A. J., and Clarke, I. J. (2006). Neuroendocrine mechanisms of innate states of attenuated responsiveness of the hypothalamo-pituitary adrenal axis to stress. Front. Neuroendocrinol. 27:285-307. doi: 10.1016/j.yfrne.2006. 06.002 
Torner, L., Karg, S., Blume, A., Kandasamy, M., Kuhn, H.-G., Winkler, J., et al. (2009). Prolactin prevents chronic stress-induced decrease of adult hippocampal neurogenesis and promotes neuronal fate. J. Neurosci. 29, 1826-1833. doi 10.1523/JNEUROSCI.3178-08.2009

Torner, L., and Neumann, I. D. (2002). The brain prolactin system: involvement in stress response adaptations in lactation. Stress 5, 249-257. doi: $10.1080 / 1025389021000048638$

Torner, L., Toschi, N., Nava, G., Clapp, C., and Neumann, I. D. (2002). Increased hypothalamic expression of prolactin in lactation: involvement in behavioural and neuroendocrine stress responses. Eur. J. Neurosci. 15, 1381-1389. doi: 10.1046/j.1460-9568.2002.01965.x

Toufexis, D. J., Thrivikraman, K. V., Plotsky, P. M., Morilak, D. A., Huang, N., and Walker, C. D. (1998). Reduced noradrenergic tone to the hypothalamic paraventricular nucleus contributes to the stress hyporesponsiveness of lactation. $J$. Neuroendocrinol. 10, 417-427. doi: 10.1046/j.1365-2826.1998.00223.x

Toufexis, D. J., and Walker, C. D. (1996). Noradrenergic facilitation of the adrenocorticotropin response to stress is absent during lactation in the rat. Brain Res. 737, 71-77. doi: 10.1016/0006-8993(96)00627-0

Vanoye-Carlo, A., Morales, T., Ramos, E., Mendoza-Rodríguez, A., and Cerbón, M. (2008). Neuroprotective effects of lactation against kainic acid treatmen in the dorsal hippocampus of the rat. Horm. Behav. 53, 112-123. doi: 10.1016/j.yhbeh.2007.09.004

Vega, C., Moreno-Carranza, B., Zamorano, M., Quintanar-Stéphano, A., Méndez, I., Thebault, S., et al. (2010). Prolactin promotes oxytocin and vasopressin release by activating neuronal nitric oxide synthase in the supraoptic and paraventricular nuclei. Am. J. Physiol. Regul. Integr. Comp. Physiol. 299, R1701-R1708. doi: 10.1152/ajpregu.00575.2010

Vukusic, S., Hutchinson, M., Hours, M., Moreau, T., Cortinovis-Tourniaire, P., Adeleine, P., et al. (2004). Pregnancy and multiple sclerosis (the PRIMS study): clinical predictors of post-partum relapse. Brain 127, 1353-1360. doi: 10.1093/brain/awh152

Walker, C. D., Lightman, S. L., Steele, M. K., and Dallman, M. F. (1992). Suckling is a persistent stimulus to the adrenocortical system of the rat. Endocrinology 130, 115-125. doi: 10.1210/en.130.1.115

Walker, C. D., Toufexis, D. J., and Burlet, A. (2001). Hypothalamic and limbic expression of $\mathrm{CRF}$ and vasopressin during lactation: implications for the control of ACTH secretion and stress hyporesponsiveness. Prog. Brain Res. 133, 99-110. doi: 10.1016/S0079-6123(01)33008-X

Walker, T. L., Vukovic, J., Koudijs, M. M., Blackmore, D. G., Mackay, E. W., Sykes, A. M., et al. (2012). Prolactin stimulates precursor cells in the adult mouse hippocampus. PLoS ONE 7:e44371. doi: 10.1371/journal.pone.0044371

Wang, J. M., Liu, L., Irwin, R. W., Chen, S., and Brinton, R. D. (2008). Regenerative potential of allopregnanolone. Brain Res. Rev. 57, 398-409. doi 10.1016/j.brainresrev.2007.08.010

Watanabe, M., Iwatani, Y., Hidaka, Y., Mitsuda, N., and Amino, N. (1996). Changes in soluble CD4 and CD8 proteins in healthy pregnant and postpartum women. Am. J. Reprod. Immunol. 36, 220-227. doi: 10.1111/j.1600-0897.1996.tb00167.x
Watanabe, M., Iwatani, Y., Kaneda, T., Hidaka, Y., Mitsuda, N., Morimoto, Y., et al. (1997). Changes in T, B, and NK lymphocyte subsets during and after normal pregnancy. Am. J. Reprod. Immunol. 37, 368-377. doi: 10.1111/j.16000897.1997.tb00246.x

Wigger, A., Lörscher, P., Oehler, I., Keck, M. E., Naruo, T., and Neumann, I. D. (1999). Nonresponsiveness of the rat hypothalamopituitary-adrenocortical axis to parturition-related events: inhibitory action of endogenous opioids. Endocrinology 140, 2843-2849. doi: 10.1210/en.140. 6.2843

Windle, R. J., Brady, M. M., Kunanandam, T., Da Costa, A. P., Wilson, B. C., Harbuz, M., et al. (1997a). Reduced response of the hypothalamo-pituitary-adrenal axis to alphal-agonist stimulation during lactation. Endocrinology 138, 3741-3748. doi: 10.1210/en.138.9.3741

Windle, R. J., Wood, S., Shanks, N., Perks, P., Conde, G. L., da Costa, A. P., et al. (1997b). Endocrine and behavioural responses to noise stress: comparison of virgin and lactating female rats during non-disrupted maternal activity. J. Neuroendocrinol. 9, 407-414. doi: 10.1046/j.1365-2826.1997.00587.x

Windle, R. J., Kershaw, Y. M., Shanks, N., Wood, S. A., Lightman, S. L., and Ingram, C. D. (2004). Oxytocin attenuates stress-induced c-fos mRNA expression in specific forebrain regions associated with modulation of hypothalamo-pituitaryadrenal activity. J. Neurosci. 24, 2974-2982. doi: 10.1523/JNEUROSCI.343203.2004

Windle, R. J., Wood, S. A., Kershaw, Y. M., Lightman, S. L., and Ingram, C. D. (2013). Adaptive changes in basal and stress-induced HPA activity in lactating and post-lactating female rats. Endocrinology 154, 749-761. doi: 10.1210/en. 2012-1779

Wintrip, N., Nance, D. M., and Wilkinson, M. (1997). The effect of lactation on induced Fos-like immunoreactivity in the rat hypothalamic paraventricular nucleus. Brain Res. 754, 113-120. doi: 10.1016/S0006-8993(97) 00063-2

Conflict of Interest Statement: The authors declare that the research was conducted in the absence of any commercial or financial relationships that could be construed as a potential conflict of interest.

Received: 30 May 2013; paper pending published: 19 August 2013; accepted: 25 December 2013; published online: 17 January 2014.

Citation: Monasterio N, Vergara E and Morales T (2014) Hormonal influences on neuroimmune responses in the CNS of females. Front. Integr. Neurosci. 7:110. doi: 10.3389/fnint.2013.00110

This article was submitted to the journal Frontiers in Integrative Neuroscience.

Copyright (c) 2014 Monasterio, Vergara and Morales. This is an open-access article distributed under the terms of the Creative Commons Attribution License (CC BY). The use, distribution or reproduction in other forums is permitted, provided the original author(s) or licensor are credited and that the original publication in this journal is cited, in accordance with accepted academic practice. No use, distribution or reproduction is permitted which does not comply with these terms. 